Janusz Dąbrowski*

\title{
Optimization of Setting out Controls in Aspect of Stakeout Accuracy of Engineering Objects**
}

\section{Introduction}

Geodetic network optimization can be carried out by taking into account the three main design criteria, which are contained in [11, 13]. Stages of designing and establishing optimal geodetic networks for the stated purposes have been presented in [6].

The authors of $[5,14,15]$, in their studies, focus attention on the selection of appropriate weights for observations in geodetic networks. One way of geodetic network optimization is the strategy of achieving balance in observations and this has been developed by Kampmann [8] and [9] and by Caspary [4]. Another geodetic network optimization method which can be used in establishing the network has been presented by Hekimoglu [7].

The problem of optimizing geodetic networks is closely related to the internal and external network reliability. The theory of network reliability is the subject of many scientific papers, and the precursors of these studies are Baarda [1-3] and Pope [10]. Significant achievements in this area can be found in works by Prószyński [11] and [12].

This work concerns a geodetic network being a setting out control, thus an essential criterion of its optimization is the limited construction tolerance, which refers to elements of the layout plan. In the optimization process the structure of the control and accuracy of surveys of its elements are taken into account. In these considerations, modern surveying instruments of the highest accuracy, the optimal number of observations that can be taken in the field, and the established accuracy of the estimation process in reference to the permissible construction tolerances of objects being staked out, are taken into account.

* AGH University of Science and Technology, Faculty of Mining Surveying and Environmental Engineering, Department of Geomatics, Krakow, Poland

** The paper is the result of studies carried out within the statutory research no. 11.11.150.006 in the Department of Geomatics, AGH University of Science and Technology, Krakow. 
On the basis of the covariance matrix for estimated coordinates of points of a setting out control a global inaccuracy parameter has been defined. The parameter takes into account the construction of the control and inaccuracy of measurements of its all elements. The global parameter of the control inaccuracy is directly connected with construction tolerances for objects intended for setting out in the field. To solve above problem two orthogonal functions $F_{d}$ and $F_{k}$ have been defined. These functions tie all points of the setting out control. Covariance matrix of these functions $\operatorname{cov}\left(F_{d^{\prime}} F_{k}\right)$ transformed onto parameters of the constant probability density ellipse illustrates the global inaccuracy of the considered setting out control. On the basis of an invariant of this matrix it is possible to select the optimal variant of the measuring designed setting out control which assures the setting out process within the permissible value of a construction tolerance.

The idea behind this article is an attempt to define an index of inaccuracy of a setting out control on the basis on the variance-covariance matrix.

\section{Construction Tolerances for Selected Structures}

Setting out a layout plan (defining urban and architectural development of an investment area) in the field relies on determining the spatial position of points representing major axes or other buildings' elements which are the contents of the layout plan. Setting out a layout plan is always carried out with reference to the previously established setting out control represented by a set of points determined with appropriate accuracy and marked up in the field.

The accuracy of determination of coordinates of points of a local control for setting out should be referenced to construction tolerances of objects covered by the layout plan. Input parameters, defining required accuracy of surveying works, are values of the limited construction tolerances (GT) which are presented in professional instructions. These tolerances are the permissible differences in horizontal and vertical positions between dimensions of a set aut object and the designed one or permissible deviations from the plumb line or inclination to a horizontal plane. The values of these tolerances should be taken into account whilst designing and establishing a local control for setting out.

Permissible values of the limited tolerances $(G T)$ are dependent on the type of a set out object, its construction and dimensions, thus they may take values from the following intervals [6]:

- deviations of distances between the axes of skeletal structures and reinforced concrete constructions should be within the interval from $3 \mathrm{~mm}$ to $7 \mathrm{~mm}$,

- displacement of anchor screws and steel or reinforced concrete pillars should be between $5 \mathrm{~mm}$ and $10 \mathrm{~mm}$,

- deviations of spacing of girders and trusses' axes and load-bearing beams should be in the range from $5 \mathrm{~mm}$ to $20 \mathrm{~mm}$, 
- deviations from the designed heights of foundations and floors, depending on the type of a construction, should be in the range from $2 \mathrm{~mm}$ to $10 \mathrm{~mm}$,

- deflection of pillars' axes from the plumb line, in terms of their height $(H)$, should be in the range from 1:250 $H$ to $1: 1000 H$,

- change of inclination of the supporting plane to the level, depending on the type of a construction, should be within the range from $1 / 250$ to $1 / 1000$.

In order to assess the inaccuracy of a setting out control a covariance matrix for the estimated coordinates of points may be used. This covariance matrix serves as a tool for determining standard deviations for particular control elements, i.e. local measures of inaccuracy for distances and directions of selected sides.

\section{Connection between the Accuracy of a Setting out Control and Construction Tolerance}

If the final effect of the setting out process is to be determination in space a position of an element of a building or its dimension then the deviation caused by surveying works $G T_{G}$ should fulfill the condition:

$$
G T_{G} \leq t \cdot G T
$$

where the parameter $t$ determines which part of the limited tolerance (GT) may go towards the deviation caused by surveying works.

The value of the parameter $t$ is set depending on the way of division of the limited tolerance into $G T_{B}$ part resulting from inaccuracy of construction and assembly works and $G T_{G}$ part due to inaccuracy of surveying works. Assuming a random model of division of tolerance GT into two equal parts:

$$
(G T)^{2}=\left(G T_{B}\right)^{2}+\left(G T_{G}\right)^{2}
$$

one obtains:

$$
G T_{B}=G T_{G} \cong 0.7 G T
$$

This means that for such a model of tolerance the value of the parameter $t$ will be on the level of 0.7 .

Deviation of surveying works $G T_{G}=0.7 G T$ consists of inaccuracy of determining points of a setting out control and inaccuracy of stakeout surveys. Assuming the simpliest random model of division of $G T_{G}$ into two equal parts, due to the inaccuracy of a setting out frame $G T_{O}$ and stakeout surveys $G T_{T^{\prime}}$ then:

$$
G T_{O}=G T_{T} \cong 0.7 G T_{G}
$$


Taking into consideration the relation (4) one may write the final formula for the limited deviation of determining spatial location of a building's element caused by inaccuracy of determination of setting out control in the form:

$$
G T_{O} \cong 0.7 \cdot 0.7 G T \cong 0.5 G T
$$

Deviation due to inaccuracy in determination of points of the setting out frame, fulfilling the condition $G T_{O} \leq 0.5 T G$ will be used to set a confidence interval for inaccuracy of determination of points of the setting out control.

In order to formulate a confidence interval for $G T_{O}$ a model in which the random variable $G T_{O}=\Upsilon$ has a normal distribution $N(\mu, \sigma)$ with both the expected value and the standard deviation unknown parametres of this distribution. Statistics:

$$
\begin{gathered}
T=\frac{\hat{Y}-\mu}{\sigma(\hat{Y})} \\
\text { for } \mu=0 \text { and } \sigma(\hat{Y})=\sqrt{\frac{\sum_{i}\left(Y_{i}-\hat{Y}\right)^{2}}{n(n-u)}}
\end{gathered}
$$

has Student's distribution with $v=(n-u)$ degrees of freedom. Because the distribution of this statistics depends only on degrees of freedom, hence, it may be used to construct a confidence interval for the most probable value of $G T_{O}$ represented by the expected value $\mu$.

The length of a symmetrical confidence interval for the estimated value of $G T_{O}=Y$ is dependent on the standard deviation of the expected value $\sigma[\hat{Y}]$, a coefficient of significance level and numbers of degrees of freedom $v$, thus it may be written as following formula:

$$
y_{\alpha}=2 t\left(1-\frac{\alpha}{2} ; v\right) \times \sigma[\hat{Y}]
$$

Relation (7) expresses a condition that the global interval inaccuracy of determining coordinates of points of a setting out control should be equal to the doubled value of Student't $t$ distribution quantile with a taken confidence level $1-\alpha$ and $v$ degrees of freedom, multiplied by the standard deviation of the random variable equal $G T_{O} \leq 0.5 G T$.

In order to fulfill the condition expressed by (7) it is necessary to work out an algorithm serving for the global assessment of setting out frame uncertainty, which in a geometrical interpretation will represent the global uncertainty of determination of relative position of points in this frame. 


\section{Orthogonal Functions for Determination the Global Inaccuracy Index of a Setting out Control}

To stake out field points representing elements of a layout plan any combinations of points of the setting out control may be selected, this means, that reference points for setting out purposes may be adjacent or opposite points of the frame, which very often are not tied by direct surveys. To define a function that includes the global inaccuracy of determining the relative positions of control points one must use all binary combinations linking each point with all other points of the control.

For this purpose two orthogonal functions, $F_{d}$ and $F_{k^{\prime}}$ have been proposed. The functions includes the geometric position of each point with respect to all other points of the control. The function $F_{d}$ is the sum of projections of partial coordinates $d x$ and $d y$ of each point in the network on all directions determined with respect to all ruemaining points. Hence, partial coordinates $d x$ will be multiplied by cosines of azimuths of considered directions and partial coordinates $d y$ will be multiplied by sines of these azimuths. But the function $F_{k}$ is orthogonal to $F_{d^{\prime}}$, hence, it represents the sum of projections of increments $d x$ and $d y$ of each point of the network on orthogonal in the relation directions to all directions considered for the function $F_{d}$.

Folmulas for $F_{d}$ and $F_{k}$ may be written:

$$
\begin{gathered}
F_{d}=d x_{1} \sum_{i=1}^{n} \cos \alpha_{1-i}+d y_{1} \sum_{i=1}^{n} \sin \alpha_{1-i}+\ldots+d x_{n} \sum_{i=1}^{n} \cos \alpha_{n-1}+d y_{n} \sum_{i=1}^{n} \sin \alpha_{n-i} \\
F_{k}=d x_{1} \sum_{i=1}^{n}\left(-\sin \alpha_{1-i}\right)+d y_{1} \sum_{i=1}^{n} \cos \alpha_{1-i}+\ldots+d x_{n} \sum_{i=1}^{n}\left(-\sin \alpha_{n-1}\right)+d y_{1} \sum_{i=1}^{n} \cos \alpha_{n-1}
\end{gathered}
$$

where quantities $\alpha_{k-i}$ denote azimuths of segments determined by a fixed point with the index $k$ to every other point in the frame with the index $i$. If $k=i$ then $\alpha_{k-i}=0$ because instead of a segment a point is identified.

If the partial coordinates $d x$ and $d y$ are represented by their standard deviations then the function $F_{d}$ will be representing an interval measure of inaccuracy in distance determination between all binary links in the network. $F_{k}$ will be presenting an interval measure of inaccuracy of azimuth determination denoted by all binary combinations of points in the considered network.

Analyzing the functions (8) and (9) it can be seen that for points of a setting out control whose coordinates will be determined by measuring a set of directions spaced approximately evenly in different quadrants of a circle, the expressions under the summation symbol will have different signs and will be mutually reducing, hence the coefficients standing by $d x$ and $d y$ will be taking values close to zero.

In cases where the set of directions are concentrated in only one quadrant of a circle, the components of sums of sines and cosines of azimuths will have the same signs, and the values of the coefficients will take values much greater than 1 . 
From the above analysis a conclusion may be drawn that the values of coefficients occurring in the functions $F_{d}$ and $F_{k}$ have the main influence on the global inaccuracy of determination of relative position of points in the setting out control.

\section{Notational Convention and Formulas to Estimate Coordinates of Points of Geodetic Networks}

In the adjustment of geodetic networks, angular and linear observations as well as coordinates of points (GPS) are used. These observations will be denoted as $L_{1}, L_{2}, \ldots, L_{n}$. These quantities are correlated by a matrix $\mathbf{G}$ and their expected values may be described by linear models AX. The parameters being estimated are the coordinates of points of geodetic network $X_{1}, X_{2}, \ldots, X_{n}$, vector of residuals $\delta$ and the coefficient of variance. $\sigma^{2}$

After applying the least squares method to such defined observational model of a setting out control and introducing the weight matrix $\mathbf{P}=\mathbf{G}^{-1}$ formulas for the estimation of parameters are obtained.

The estimator of the vector $\mathbf{X}$ representing small corrections to approximate coordinates of points of the control is expressed as:

$$
\hat{X}=\left(\mathbf{A}^{T} \mathbf{P A}\right)^{-1} \mathbf{A}^{T} \mathbf{P L}=\mathbf{A}^{+} \mathbf{L}
$$

Formula (10) defines the unbiased estimator of the vector $\mathbf{X}$, hence the covariance matrix of this vector is expressed:

$$
\operatorname{cov}[\hat{X}]=\hat{\sigma}^{2}(\mathbf{A P A})^{-1}
$$

Estimator of the variance $\sigma^{2}$ coefficient is being determined on the basis of the following formula:

$$
\hat{\sigma}^{2}=\frac{\mathbf{L}^{T} \mathbf{P L}-\hat{\mathbf{X}}^{T} \mathbf{A}^{T} \mathbf{P L}}{n-k}
$$

where $k=R(\mathbf{A})$.

\section{Global Inaccuracy of Determining the Relative Position of Points in a Setting out Control}

The functions defined by eqs. (8) and (9) constitute the base for a global assessment of inaccuracy of a setting out control. Coordinates of points are estimated by means of the formula (10) and their covariance matrix by means of eqs. (11) and (12). 
On the basis of analysis of variance for the functions (8) and (9) a covariance matrix for $F_{d}$ and $F_{k}$ may be obtained:

$$
\begin{aligned}
& \operatorname{cov}\left[\begin{array}{cc}
V\left(F_{d}\right) & \operatorname{cov}\left(F_{d}, F_{k}\right) \\
\operatorname{cov}\left(F_{d}, F_{k}\right) & V\left(F_{k}\right)
\end{array}\right]= \\
& =\left[\begin{array}{cc}
\sum_{i=1}^{n} \cos \alpha_{1-i} & -\sum_{i=1}^{n} \sin \alpha_{1-i} \\
\sum_{i=1}^{n} \sin \alpha_{1-i} & \sum_{i=1}^{n} \cos \alpha_{1-i} \\
\sum_{i=1}^{n} \cos \alpha_{2-i} & -\sum_{i=1}^{n} \sin \alpha_{2-i} \\
\sum_{i=1}^{n} \sin \alpha_{2-i} & \sum_{i=1}^{n} \cos \alpha_{2-i} \\
\ldots \ldots \ldots & \ldots \ldots \ldots . \\
\ldots \ldots \ldots & \ldots \ldots \ldots . \\
\sum_{i=1}^{n} \cos \alpha_{n-i} & -\sum_{i=1}^{n} \sin \alpha_{n-i} \\
\sum_{i=1}^{n} \sin \alpha_{n-i} & \sum_{i=1}^{n} \cos \alpha_{n-i}
\end{array}\right] \operatorname{cov}[\hat{X}]\left[\begin{array}{cc}
\sum_{i=1}^{n} \cos \alpha_{1-i} & -\sum_{i=1}^{n} \sin \alpha_{1-i} \\
\sum_{i=1}^{n} \sin \alpha_{1-i} & \sum_{i=1}^{n} \cos \alpha_{1-i} \\
\sum_{i=1}^{n} \cos \alpha_{2-i} & -\sum_{i=1}^{n} \sin \alpha_{2-i} \\
\sum_{i=1}^{n} \sin \alpha_{2-i} & \sum_{i=1}^{n} \cos \alpha_{2-i} \\
\ldots \ldots \ldots . . & \ldots \ldots \ldots . . \\
\ldots \ldots \ldots . & \ldots \ldots \ldots . . \\
\sum_{i=1}^{n} \cos \alpha_{n-i} & -\sum_{i=1}^{n} \sin \alpha_{n-i} \\
\sum_{i=1}^{n} \sin \alpha_{n-i} & \sum_{i=1}^{n} \cos \alpha_{n-i}
\end{array}\right]
\end{aligned}
$$

The matrix in the formula (13) contains information on the interval inaccuracy of determination of points in all binary combinations.

The ellipse of constant density probability on the level of the mean error for the considered functions $F_{d}$ and $F_{k}$ (having a strict geometrical interpretation) may be the image of this inaccuracy.

On the basis of the covariance matrix (13) semiaxes A and B of the constant density probability ellipse are determined as follows:

$$
\begin{aligned}
& {\left[\begin{array}{cc}
V\left(F_{d}\right) & \operatorname{cov}\left(F_{d}, F_{k}\right) \\
\operatorname{cov}\left(F_{d}, F_{k}\right) & V\left(F_{k}\right)
\end{array}\right] \Rightarrow} \\
& \Rightarrow\left\{\begin{array}{l}
A^{2} \\
B^{2}
\end{array}\right\}=\frac{V\left(F_{d}\right)+V\left(F_{k}\right)}{2} \pm \sqrt{\left[\frac{V\left(F_{d}\right)-V\left(F_{k}\right)}{2}\right]^{2}+\left[\operatorname{cov}\left(F_{d}, F_{k}\right)\right]^{2}}
\end{aligned}
$$

Azimuth of the semi-major axis $\mathbf{A}$ of this ellipse is determined by the formula:

$$
\operatorname{arctg} 2 \alpha_{A}=\frac{2 \operatorname{cov}\left(F_{d}, F_{k}\right)}{V\left(F_{d}\right)-V\left(F_{k}\right)}
$$


The parameters of the ellipse determined by (14) and (15) illustrated graphically in a suitable scale may constitute the base for the global assessment of inaccuracy for a designed or already established control.

The length of the semiaxis $\mathbf{A}$ of the ellipse represents the global inaccuracy of determination of point position in the azimuth $\alpha_{A}$, thus, in the direction in which the network is determined in the weakest way.

The parameters of the constant density probability ellipse may also be the base for defining a one - dimensional index of the interval inaccuracy of the control. The value of this index should be based on the area of the ellipse i.e. on the product of the lengths of its axes. After some manipulations the following formula has been obtained:

$$
A^{2} \cdot B^{2}=V\left[F_{d}\right] \cdot V\left[F_{k}\right]-\operatorname{cov}^{2}\left[F_{d}, F_{k}\right]
$$

To give a practical dimension to the above relation, the area of the ellipse is replaced by the area of a circle whose radius $R_{G}$ will represent the global interval inaccuracy of determination the coordinates of all points considered in the control i.e.:

$$
R_{G}=\sqrt[4]{V\left[F_{d}\right] \cdot V\left[F_{k}\right]-\operatorname{cov}^{2}\left[F_{d}, F_{k}\right]}
$$

It is worth noting that the expression under the fourth root symbol is the determinant of the covariance matrix defined by the formula (13) which is the invariant of transformation of coordinates of points in the setting out frame.

If, in place of $y_{\alpha^{\prime}}$ in the formula (7) the interval inaccuracy of determination the coordinates of all points considered in the frame is substituted and if $\sigma(\hat{Y})$ is replaced by $G T_{O}=0.5 G T$ then a condition to be fulfilled in order not to exceed the established part of the limiting construction tolerance due to the setting out control is obtained:

$$
R_{G} \leq 2 t\left(1-\frac{\alpha}{2} ; v\right) \cdot 0.5 G T \Rightarrow R_{G} \leq t\left(1-\frac{\alpha}{2} ; v\right) G T
$$

From the above condition results that the global interval inaccuracy of determination of coordinates of all points considered in the control should be less than the limiting construction tolerance multiplied by a quantile of Student's $t$ distribution.

This quantile should be taken for the confidence level equal to $1-\alpha=0.95$ and $v=n-u$ degrees of freedom i.e. equal to the number of additional observations in the considered network. 


\section{Verification of Formulas} on Global Inaccuracy of Determination of Setting out Control Points in the Conection with Construction Tolerance Numerical Example

Verification of the formulas will be presented on an example of a setting out frame in the form of four points, shown in Figure 1, approximate coordinates of these points in a local frame are given in Table 1.

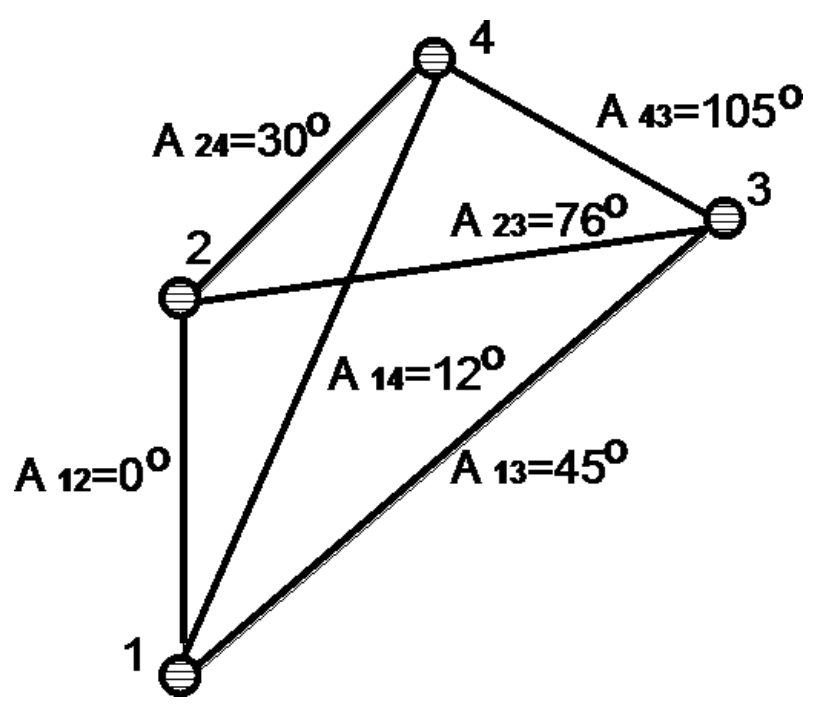

Fig. 1. Sketch of the setting out control

Table 1. Approximate coordinates of points in a local frame

\begin{tabular}{|c|c|c||}
\hline Point number & $X[\mathrm{~m}]$ & $Y[\mathrm{~m}]$ \\
\hline \hline 1 & 200.10 & 50.40 \\
\hline 2 & 320.20 & 50.42 \\
\hline 3 & 360.26 & 210.46 \\
\hline 4 & 389.72 & 90.48 \\
\hline
\end{tabular}

Measurements of six distances and four angles were the base for the estimation process according to formula (10) its coordinetes of prints and according formulas (11) and (12) their covariation matrices.

The point number 4 was assumed fixed hence the number of estimated coordinates of the points is $2 \cdot 3=6$. 
The resulting covariance matrix was determined for $(10-6)=4$ degrees of freedom and the values of its entries are shown in the following matrix:

$$
\operatorname{cov}[\hat{X}]=\left[\begin{array}{cccccc}
48.42 & 12.84 & 8.43 & -4.24 & 7.98 & 11.40 \\
12.84 & 62.20 & 2.14 & 0.20 & -4.40 & 7.10 \\
8.43 & 2.14 & 38.64 & 6.36 & 7.42 & 9.20 \\
-4.24 & 0.20 & 6.36 & 44.76 & 3.52 & -5.22 \\
7.98 & -4.40 & 742 & 3.52 & 68.38 & 7.66 \\
11.40 & 7.10 & 9.20 & -5.22 & 7.66 & 56.64
\end{array}\right]\left[\mathrm{mm}^{2}\right]
$$

Coefficients of functions $F_{d}$ and $F_{k}$ determined for points 1, 2 and 3 of considered control take the following form:

$$
\left[F_{d}\right]=\left[\begin{array}{c}
2.685 \\
0.914 \\
-0.134 \\
1.500 \\
-0.207 \\
-2.573
\end{array}\right] ; \quad\left[F_{k}\right]=\left[\begin{array}{c}
-0.914 \\
2.685 \\
-1.500 \\
-0.134 \\
2.573 \\
-0.207
\end{array}\right] ; \quad \vec{F}_{d} \cdot \vec{F}_{k}=0
$$

After applying formula (13) the following covariance matrix has been obtained:

$$
\operatorname{cov}\left[F_{d}, F_{k}\right]=\left[\begin{array}{cc}
V\left(F_{d}\right) & \operatorname{cov}\left(F_{d}, F_{k}\right) \\
\operatorname{cov}\left(F_{d}, F_{k}\right) & V\left(F_{k}\right)
\end{array}\right]=\left[\begin{array}{cc}
755.8536 & 83.9028 \\
83.9028 & 814.5015
\end{array}\right]\left[\mathrm{mm}^{2}\right] .
$$

The determinant of this matrix and its fourth root take the following values:

$$
\operatorname{det}\left[\operatorname{cov}\left[F_{d}, F_{k}\right]=608604.2027 \Rightarrow \sqrt[4]{608604.2027}=27.93 \mathrm{~mm}\right.
$$

Parameters of the constant density probability ellipse, defined by equations (14) and (15), take the values:

$$
\begin{aligned}
& \left\{\begin{array}{l}
A^{2} \\
B^{2}
\end{array}\right\}=785.18 \pm \sqrt{\left[\frac{-58.65}{2}\right]^{2}+83.90^{2}}=785.18 \pm 88.87=\left\{\begin{array}{l}
874.05 \\
696.31
\end{array}\right\}\left[\mathrm{mm}^{2}\right] \\
& \operatorname{arctg} 2 \alpha_{A}=\frac{2 \cdot 83.90}{-58.65}=-2.8610 \Rightarrow 2 \alpha_{A}=321.40^{\mathrm{g}} \Rightarrow \alpha_{A}=160.70^{\mathrm{g}} .
\end{aligned}
$$

On the basis of the above parameters a conclusion may be drawn that the global inaccuracy of the considered control is illustrated by the constant density probability 
ellipse with the semi-major axis $\mathbf{A}=29.6 \mathrm{~mm}$, semi-minor axis $\mathbf{B}=26.4 \mathrm{~mm}$. The azimuth determining a direction of the greatest global inaccuracy is equal to $\alpha=160.70$.

The radius of the circle $R_{G}$ with the area equal to the area of the ellipse will represent a global interval inaccuracy of the determination of the mutual position of points in all binary combinations in the considered control:

$$
R_{G}=\sqrt[4]{A^{2} \cdot B^{2}}=\sqrt[4]{874.06 \cdot 696.30}=27.9 \mathrm{~mm} .
$$

If the condition (18), for the confidence level $1-\alpha=0.95$ and degrees of freedom $v=4$, is used then the following accuracy criterion for the considered network will be obtained:

$$
T \geq \frac{R_{G}}{t\left(1-\frac{\alpha}{2} ; v\right)} \Rightarrow G T \geq \frac{27.93}{2.8} .
$$

Substituting all necessary quantities the final value of the criterion is given by:

$$
G T \geq 10.0 \mathrm{~mm} \text {. }
$$

A conclusion of this analysis is that the considered points of the control may be successfully used to set out objects for which the permissible construction tolerance is greater than $10.3 \mathrm{~mm}$.

If the value of this tolerance is less than $10.0 \mathrm{~mm}$ then the network should be strengthened with additional observations or the measurements should be carried out with more accurate surveying instruments and the entire procedure should be repeated afterwards.

In order to compare the stability of determination of the global inaccuracy parameters of the net, the same covariance matrix has been used but with the assumption that only two points 1 and 2 will be taking part in the setting out process.

Coefficient of function $F_{d}$ and $F_{k}$ for the current case take the following form:

$$
\left.\left[F_{d}\right]=\left[\begin{array}{c}
2.685 \\
0.914 \\
-0.134 \\
1.500 \\
0 \\
0
\end{array}\right] ; \quad \quad F_{k}\right]=\left[\begin{array}{c}
-0.914 \\
2.685 \\
-1.500 \\
-0.134 \\
0 \\
0
\end{array}\right] ; \quad \vec{F}_{d} \times \vec{F}_{k}=0 \text {. }
$$


Again, after applying formula (13) covariance matrix of the form has been obtained:

$$
\left[\begin{array}{cc}
V\left(F_{d}\right) & \operatorname{cov}\left(F_{d}, F_{k}\right) \\
\operatorname{cov}\left(F_{d}, F_{k}\right) & V\left(F_{k}\right)
\end{array}\right]=\left[\begin{array}{cc}
523.0395 & 72.7093 \\
72.7093 & 523.7216
\end{array}\right]\left[\mathrm{mm}^{2}\right] .
$$

The determinant of this matrix and its fourth root take the following values:

$$
\operatorname{det}\left[\operatorname{cov}\left[F_{d}, F_{k}\right]=268640.4573 \Rightarrow \sqrt[4]{268640.4573}=22.77 \mathrm{~mm}\right.
$$

Parameters of the ellipse of constant probability take the values:

$$
\begin{aligned}
& \left\{\begin{array}{l}
A^{2} \\
B^{2}
\end{array}\right\}=523.38 \pm \sqrt{\left[\frac{-0.6821}{2}\right]^{2}+72.7093^{2}}=523.38 \pm 72.71=\left\{\begin{array}{l}
596.09 \\
450.67
\end{array}\right\}\left[\mathrm{mm}^{2}\right] \\
& \operatorname{arctg} 2 \alpha_{A}=\frac{2 \cdot 72.7093}{-0.6821}=-213.1925 \Rightarrow 2 \alpha_{A}=300.30^{\mathrm{g}} \Rightarrow \alpha_{A}=150.15^{\mathrm{g}} .
\end{aligned}
$$

The radius of the circle $R_{G}$ with the area equal to the area of the ellipse will represent a global interval uncertainty of the determination of the relative position of points in all binary combinations in the net:

$$
R_{G}=\sqrt[4]{A^{2} \cdot B^{2}}=\sqrt[4]{596.09 \times 450.67}=22.77 \mathrm{~mm} .
$$

If the condition (18), for the confidence level $1-\alpha=0.95$ and degrees of freedom $v=4$, is used then the following accuracy criterion for the considered part of the net will be obtained:

$$
G T \geq \frac{22.77}{2.8} \Rightarrow G T \geq 8.13 \mathrm{~mm}
$$

On the basis of the obtained results one may conclude that the considered part of the setting out control in the form of a base determined by the points 1 and 2 may be used to set out objects for which the permissible construction tolerance is greater than $8.1 \mathrm{~mm}$.

\section{Conclusions}

The discussion presented in the paper concerning setting out control can be fully applied to the optimization within the design, measurement and estimation of 
reference networks that can be used to determine the coordinates of points representing inventoried engineering structures or to determine displacements of points of examined objects.

The purpose of optimization within the network of reference points is to minimize field measurement works by selecting appropriate elements of the network to be measured as well as the selection of adequate equipment, so that the proposed network of reference points assure acceptable tolerances of determining coordinates of points representing engineering structures or limiting inaccuracy of determination of displacement of points of examined objects.

In the formula (18) permissible tolerances of accuracy in determining the inventoried point or the limiting uncertainty in determination of the displacement vector take the role of the limited construction tolerance $(T G)$.

\section{References}

[1] Baarda W.: Statistical Concepts in Geodesy. Netherlands Geodetic Commission, Publications on Geodesy, New Series vol. 2, no. 4, Rijkscommissie voor Geodesie Delft, The Netherlands 1967.

[2] Baarda W.: A Testing Procedure for Use in Geodetic Networks. Netherlands Geodetic Commission, Publications on Geodesy, New Series vol. 2, no. 5, Rijkscommissie voor Geodesie Delft, The Netherlands 1968.

[3] Baarda W.: Measures for the accuracy of geodetic networks. [in:] International Symposium organized by the International Association of Geodesy and by the Geodetic and Geophysical Research Institute of the Hungarian Academy of Sciences, Sopron, Hungary, 4-10 July, 1977, pp. 419-436.

[4] Caspary W.: Anmerkungen zur balancierten Ausgleichung. Zeitschrift für Vermessungswesen, vol. 123, no. 8, 1998, pp. 271-272.

[5] Cross P.A.: Numerical methods in network design. [in:] Grafarend E.W., Sanso F. (eds), Optimization and design of geodetic networks, Springer, Berlin - Heidelberg - New York 1985, pp. 132-168.

[6] Czaja J.: Modele statystyczne w informacji o terenie. Wydawnictwa AGH, Kraków 1996.

[7] Hekimoglu S.: Change of the diagonal elements of the hat matrix under changing weight and changing position of an observation, Zeitschrift für Vermessungswesen, vol. 123, no. 8, 1998, pp. 266-271.

[8] Kampmann G., Krause B.: Balanced observations with a straight line fit. Bolletino di Geodesia a Science Affini, vol. 55(2), 1996, pp. 134-141.

[9] Kampmann G.: Robuste Deformationsanalyse mittels balancierter Ausgleichung. Allgemeine Vermessungs-Nachrichten, vol. 101(1), 1994, pp. 8-17. 
[10] Pope A.J.: The Statistics of Residuals and the Detection of Outliers. Geodetic Research and Development Laboratory, National Geodetic Survey, National Ocean Survey, Rockville 1976.

[11] Prószyński W.: Measuring the robustness potential of the last-squares estimation: geodetic illustration. Journal of Geodesy, vol. 71, 1997, pp. 652-659.

[12] Prószyński W.: On outlier-hiding effects in specific Gauss-Markov models: geodetic examples. Journal of Geodesy, vol. 74, 2000, pp. 581-589.

[13] Prószyński W., Kwaśniak M.: Podstawy geodezyjnego wyznaczania przemieszczeń. Pojęcia i elementy metodyki. Oficyna Wydawnicza Politechniki Warszawskiej, Warszawa 2006.

[14] Schaffrin B.: Aspects of network design. [in:] Grafarend E.W., Sanso F. (eds), Optimization and Design of Geodetic Networks, Springer, Berlin - Heidelberg New York 1985, pp. 548-597.

[15] Schmitt G.: Second order design. [in:] Grafarend E.W., Sanso F. (eds), Optimization and Design of Geodetic Networks, Springer, Berlin - Heidelberg - New York 1985, pp. 74-121. 